\title{
Hot tearing test for TIG welding of aluminum alloys: application of a stress parallel to the fusion line.
}

\author{
A. Niel ${ }^{1}$, F. Deschaux ${ }^{1}$, C. Bordreuil ${ }^{1}$, G. Fras ${ }^{1}$ - J-M. Drezet ${ }^{2}$ \\ ${ }^{1}$ Mechanics and Civil Engineering Laboratory LMGC UMR 5508, \\ University Montpellier2, Montpellier, France \\ ${ }^{2}$ Computational Materials Laboratory, Ecole Polytechnique Federale \\ Lausanne, Switzerland
}

\begin{abstract}
Defects control such as hot cracking or solidification cracking, in aluminum alloys welding is an important industrial issue and must be carefully examined. This phenomenon is a complex problem involving process, material and mechanical loading due to clamping. Several tests have been previously developed in order to characterize the material propensity to hot cracking. The purpose of the present work is to study, using a new hot cracking test and numerical simulation, the relationship between mechanical and metallurgical factors in order to better identify the parameters leading to hot tearing during welding.

The originality of the test presented here is that an external stress is applied on the test specimen parallel to the welding direction. The advantage of this test, compared to others like Varestraint test, is its simplicity and therefore its possible for an industrial use. A weld seam is made with a Tungsten Inert Gas (TIG) arc welding on a thin sheet of aluminum alloy (6061). The crack initiation occurs once steady state thermal conditions are reached. The present test enables to distinguish between the structural effects on a global scale and the microstructural effects on a local scale. Microstructure control is made possible by adjusting welding power, welding speed and sample geometries. The grain structure which is characterized by the shape, size and the growth direction and which depends on welding current and speed plays a crucial role in the crack initiation. Microstructural features are observed using high speed camera recording and post mortem micrographs. Mechanical factors are varied by adjusting the welding parameters and the applied initial stress. The relationship between welding parameters, grain morphology, and sensitivity to hot cracking are discussed. Experimental measurements and
\end{abstract}


numerical results will help to better determine global and local conditions at the onset of hot tearing and to compare those conditions using existing hot tearing criteria.

\section{Introduction}

Welding processes are extensively used to assemble components in many manufacturing industries, such as aeronautics, construction, energy and automotive. To increase their productivity, constructors try to reduce manufacturing time. This involves for welding operations an increase of welding speed. However, various defects such as hot tearing might limit this increase. Figure 1 illustrates the presence of a crack created during arc welding on a 6061 aluminum alloy.

Welding process promotes hot cracking phenomenon due to the liquid / solid phase change. Many aspects must be taken into account to reduce the cracking risk. This complex phenomenon, involving metallurgical and mechanical factors, is studied using a new test. This complex phenomenon, involving metallurgical and mechanical factors, is studied using a new test. This test, shown in Fig 4 and 6, consists to apply a horizontal tensile before welding, in the welding direction. In comparison, for example, the programmable deformation cracking (PVR) test and the controlled tensile weldability (CTW) test consists of applying a variable tensile strain during welding, not before. Metallurgical factors involved include especially the solidification range, grain size and shape, which alter the permeability of the mushy zone. This work proposes to study the interaction between the process, the material and the mechanical loading in order to reduce hot cracking sensitivity.

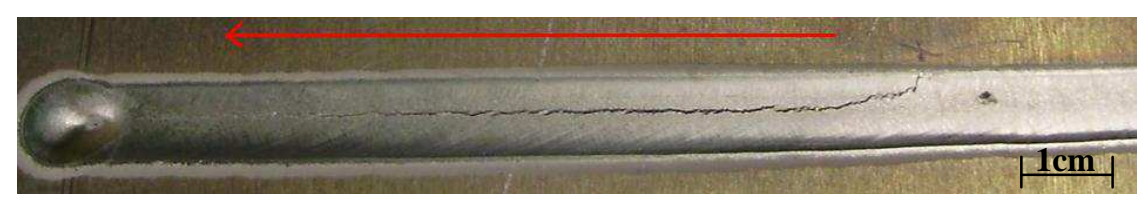

Fig. 1. Hot tearing in TIG welding.

Defects caused by welding operation are numerous and are related to metallurgical factors, welding parameters or external conditions. Among the main welding defects, hot tearing, porosity, corrosion or sticking can be quoted. Hot tearing appears at the solidification end of an alloy, and is 
commonly observed in welding. This section describes the mechanisms of hot crack initiation and the main factors responsible for this phenomenon.

During welding, components are subjected to high thermal gradients around the melting zone due to localized heat input. The solidification, zone where microstructure forms, is located at the rear of the melting zone and is bordered by two isothermal surfaces corresponding to liquidus and solidus temperatures. The mushy zone corresponds to the interval between the coherency temperature and solidus. It's a zone between percolated solid (all grains are in contact) formation and the solid coexistence of liquid and solid phases.

Figure 2 shows dendritic grain growth during solidification as a function of solid fraction. Solidification is divided in several stages (figure 2) [10]. Nucleation is the first step, solid particles nucleate in the liquid and are free to move. The material behaves like a viscous fluid with a very low tensile strength. Then, grains grow. During welding, the solidification rate is high, and the solidification front (solid-liquid interface) is dendritic. The solid fraction increases gradually and the temperature approaches the solidus. The coherency solid fraction is then reached and corresponds to first solid bridge bonding. The dendritic grains are in contact with each other and form a coherent solid skeleton. A sharp drop in permeability is then observed, due to the compact network formation. Thin liquid films between the solid grains remain and are subjected to high strains, induced by solidification shrinkage and thermal contraction of the solid. During this stage, the strain to fracture of the alloy is very weak, because the liquid can no longer flow to accommodate deformation and the solid network is not resistant enough to avoid cracking. Finally, the last stage corresponds to the coalescence solid fraction. The last isolated liquid pockets solidify, and the solid fraction tends toward one. The hot ductile solid can then resist to strain. The stress and strain to tensile failure is rising rapidly.

Brittle Temperature Range (BTR) is defined as the temperature interval corresponding to the solid fractions where the microstructure is in critical configuration. Hot tearing risk is maximal in this temperature range. The BTR corresponds to the interval between the coherency solid fraction, where the liquid does not easily circulate because of the low permeability, and the coalescence solid fraction, where the solid opposes mechanical resistance due to rise of the number of solid bridges formed. 


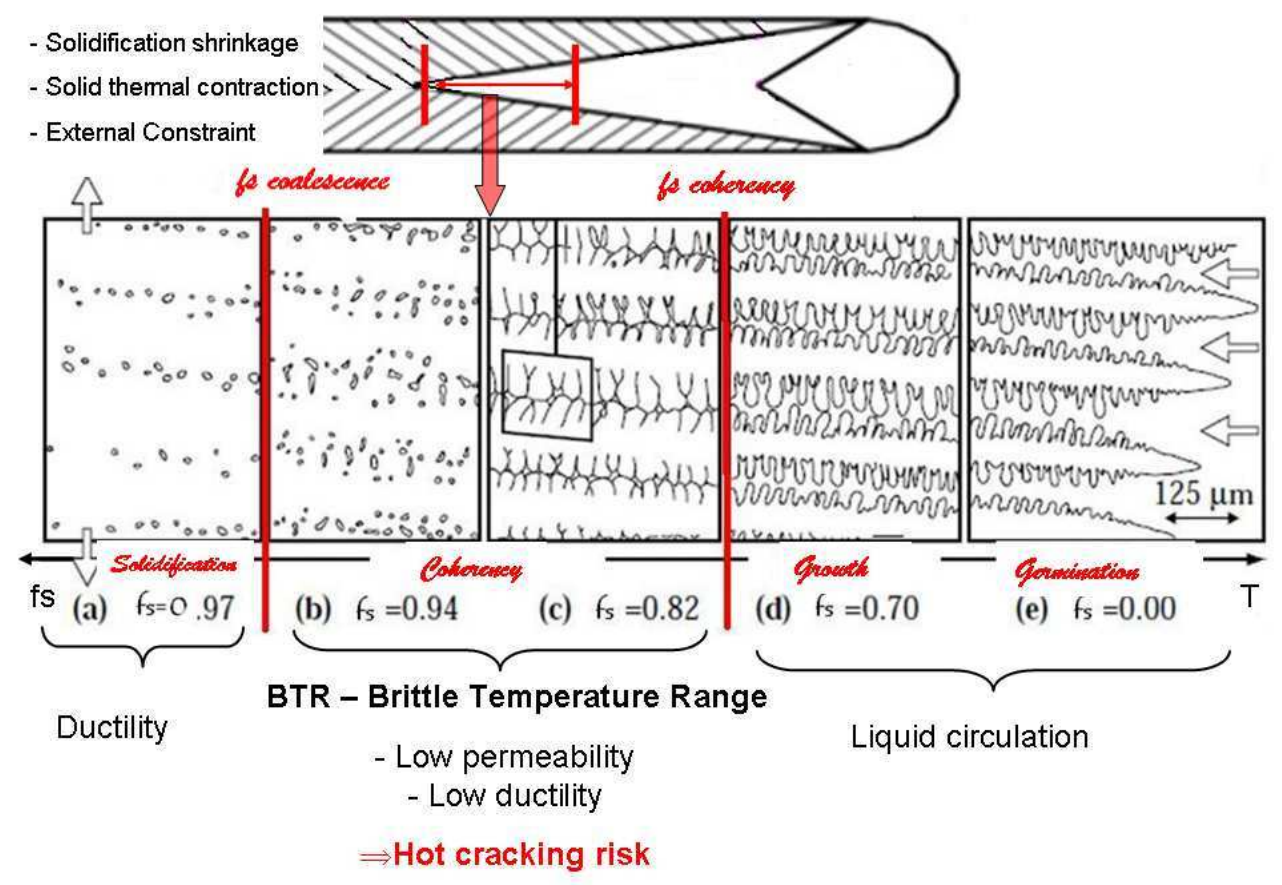

Fig. 2. Schematics of hot tearing formation in a columnar structure (from [10])

In welding, the mushy zone undergoes thermal and mechanical stresses during solidification [2]. Resulting stress and strain fields are very complex to predict due to two main factors. The first one is the result of differential shrinkage imposed by high temperature gradients. The second one is a consequence of the mechanical properties thermal dependence on different phases. All these stresses can cause liquid films to debond and/or solid bridges to break causing crack initiation.

Hot tearing is then related to process parameters, such as welding current and welding speed, but also to the composition of the material welded. Solidification conditions play an important role in susceptibility to hot cracking. The chemical composition and the solidification mode modify the quantity of residual elements with low melting point favoring the survival of residual liquid films. AA 6061, is very sensitive to hot cracking due to its wide solidification range. This alloy has structural hardening, so its high temperature behavior is complex.

Thermal contraction is the main factor of mechanical loading in the mushy zone. The thermal gradient, imposed by the process, affects solidification microstructure and heat distribution in the sheet, which change mechanical loading. From a mechanical point of view, the welding 
process leads to a non uniform distribution of temperature, combined with thermal stresses and localized plastic deformation. The thermally induced deformations (solidification shrinkage and thermal contraction) are exerted over the BTR. The non-uniformity of thermal gradient and the nonlinearity in the mechanical behavior of the material, however, increase the complexity of the problem.

The purpose of this research is to explore ways for optimizing the welding parameters with respect to hot tearing. These defects strongly affect the weld quality and then mechanical strength of welded components. This paper first presents the hot cracking phenomenon. Then an original test developed for hot tearing characterization in welding is presented. Next, experimental results achieved in TIG welding on AA6061 are analyzed. In parallel to this experimental investigation, a thermomechanical modeling of the welding operation is developed. Finally, the analysis of the microstructure influence on susceptibility to hot cracking is discussed in order to propose improvements to the welding process.

It has been seen in the previous section that crack initiation is controlled by metallurgical factors, such as solidification range, grain size and shape, mushy zone permeability, and by mechanical factors, such as stresses, strains and strain rates acting on the mushy zone. The purpose of this work is to study the interaction between mechanical and metallurgical factors in order to better identify the parameters influencing the hot tearing phenomenon in welding. The originality of this study is to try to distinguish the process influence on hot cracking by experimental and numerical results. 

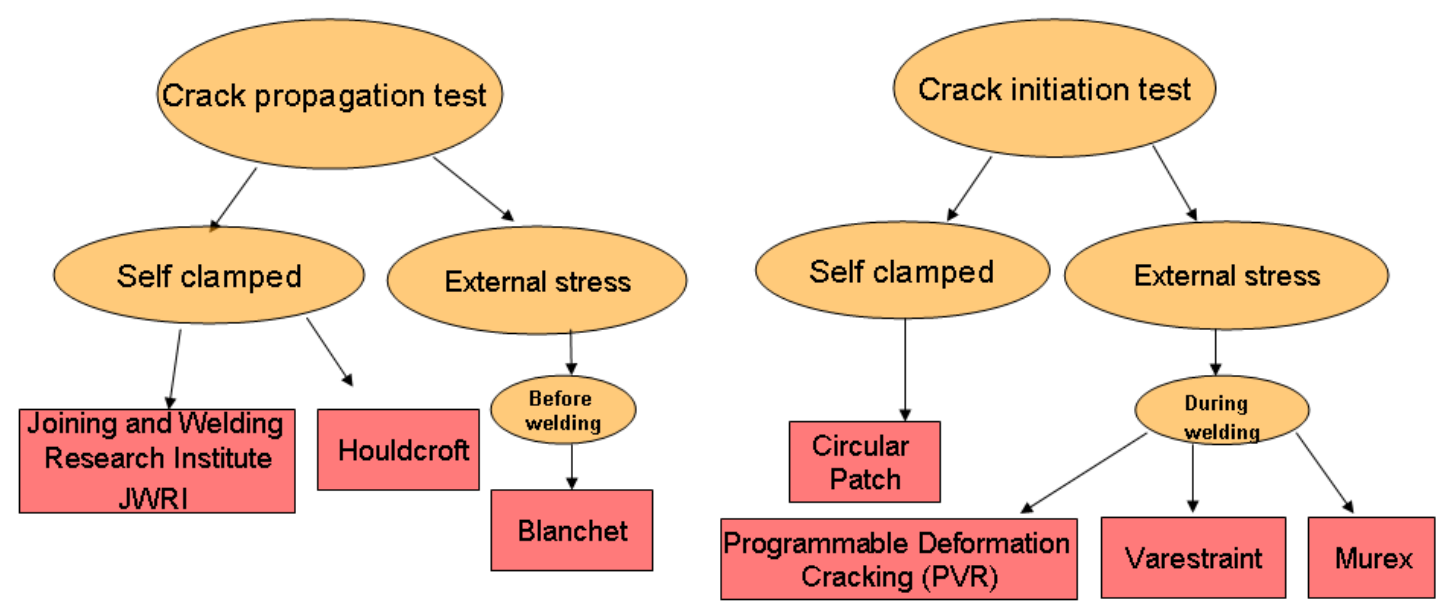

Fig. 3. Cracking test: state of art.

To assess hot cracking sensitivity of an alloy, various tests have been developed [3]. In figure 3, the existing tests have been classified into two categories: crack initiation tests (Varestraint test type) and propagation tests (Joining and Welding Research Institute: JWRI test type). The mechanical stress applied can be external to the specimen or produced by self clamping. These tests are generally difficult to interpret and implement in an industrial environment. Moreover, it is difficult to distinguish the sample geometry effects and the process effects, because cracks are the result of complex interactions between these factors. A new hot tearing test has been developed to better understand the influence of the process parameters on the sensitivity to crack initiation. It is presented here after.

\section{The experimental system}

A simple original hot tearing test for thin sheets has been developed in this study. The aim is to impose an external and controlled mechanical loading promoting cracking, to study the process and material influence on hot cracking susceptibility. This test allows the initiation of a hot tear under controlled experimental conditions. 


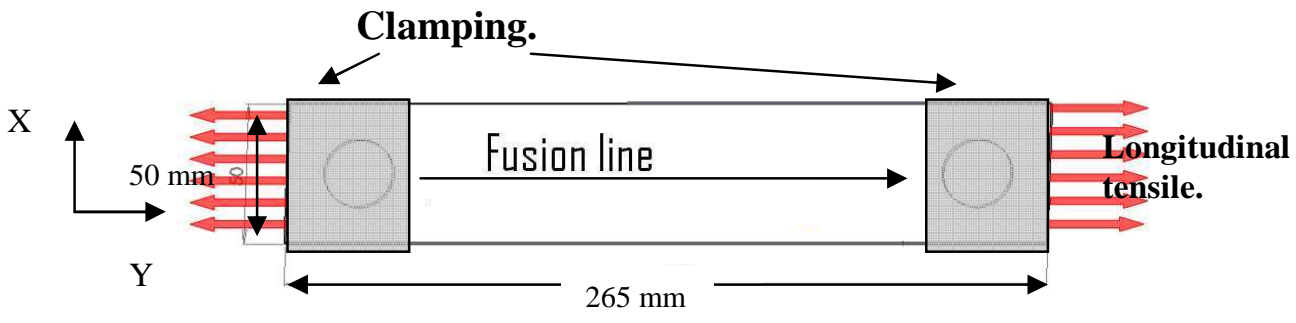

Fig. 4. Longitudinal tensile specimen with TIG weld seam.

The test originality is to promote crack initiation through a single solicitation. It involves applying a controlled tensile preload in the welding direction and not perpendicular to it as in many cracking tests, before welding without additional filler material (figure 4). During welding, the specimen is clamped on both sides between two jaws. The process used is the Tungsten Inert Gas process (TIG), also called Gas Tungsten Arc Welding (GTAW). This process uses a tungsten refractory electrode to create an electric arc, and an inert gas, generally argon, to protect metal against oxidation. A weld seam is made on a parallelepiped sheet in the longitudinal direction. Hot tearing test is placed on a mobile two axes $(\mathrm{X}$, Y) table, as depicted in figure 6. During the test, the welding arc is in a fixed position, and the sample is translated in the longitudinal direction at a constant speed. The used sheets are thin $(2.3 \mathrm{~mm})$ and the samples, cut with a water jet machine, are $265 \times 50 \mathrm{~mm}^{2}$ in size. Microstructure variation obtained by changing the welding speed and changes of tensile initial stress, allow us to investigate structural effects on hot tearing.

The test advantage, compared to other hot tearing tests such as the Varestraint test, is its simplicity which is interesting for an industrial use. In addition, the simple sample geometry and boundary conditions make the $2 \mathrm{D}$ numerical simulation of the test easy. The mechanical condition applied to the plate is assumed flat. Indeed, measurements using strain gauges showed that the tensile stress imposed by the test and the welding cycle generate plane strain. The used sheets are thin $(2.3 \mathrm{~mm})$ and permit 2D modeling. In this test, control microstructure is possible by adjusting thermal cycle, depending on welding power, speed and sample size. Microstructural characteristics are observed using high speed camera recording and post mortem analysis. The high speed camera is in static position to observe the weld pool rear. 


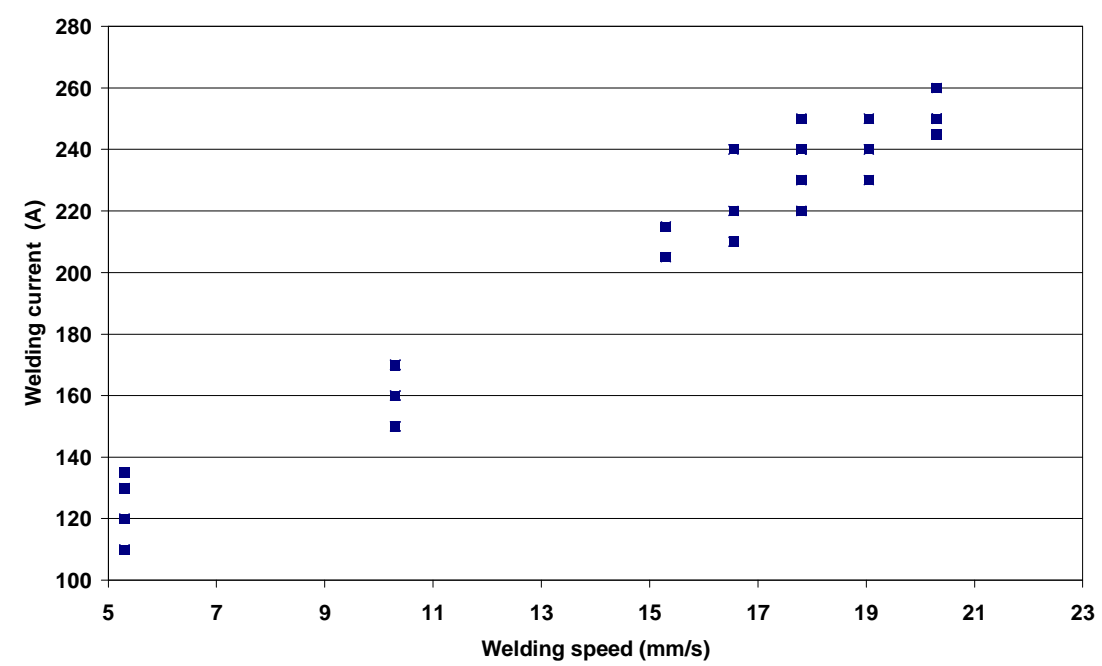

Fig. 5. Welding condition of the first test campaign.

Six welding speeds, between $5 \mathrm{~mm} / \mathrm{s}$ and $20 \mathrm{~mm} / \mathrm{s}$, were studied. Four tests were conducted for each speed by varying the welding current in the range [130A, 260A]. The exact welding condition is show in figure 5. The alternative current in TIG welding is necessary on aluminum. At each cycle, there is a polarity reversal that breaks the alumina layer formed on the surface. The ratio welding current / welding speed is set to have full penetration. A $3 \mathrm{~mm}$ arc length is imposed for all the welding tests, which corresponds to a welding voltage of about $10 \mathrm{~V}$. In total, 24 samples have been studied with varying welding parameters and a pre- stress of 200 $\mathrm{MPa}(0,8 \mathrm{~mm}$ initial displacement imposed at the sample end). Note that the welding speed range is rather high for TIG welding process and that the weld seam is started far away from the jaws so that the initial stress is uniaxial. 


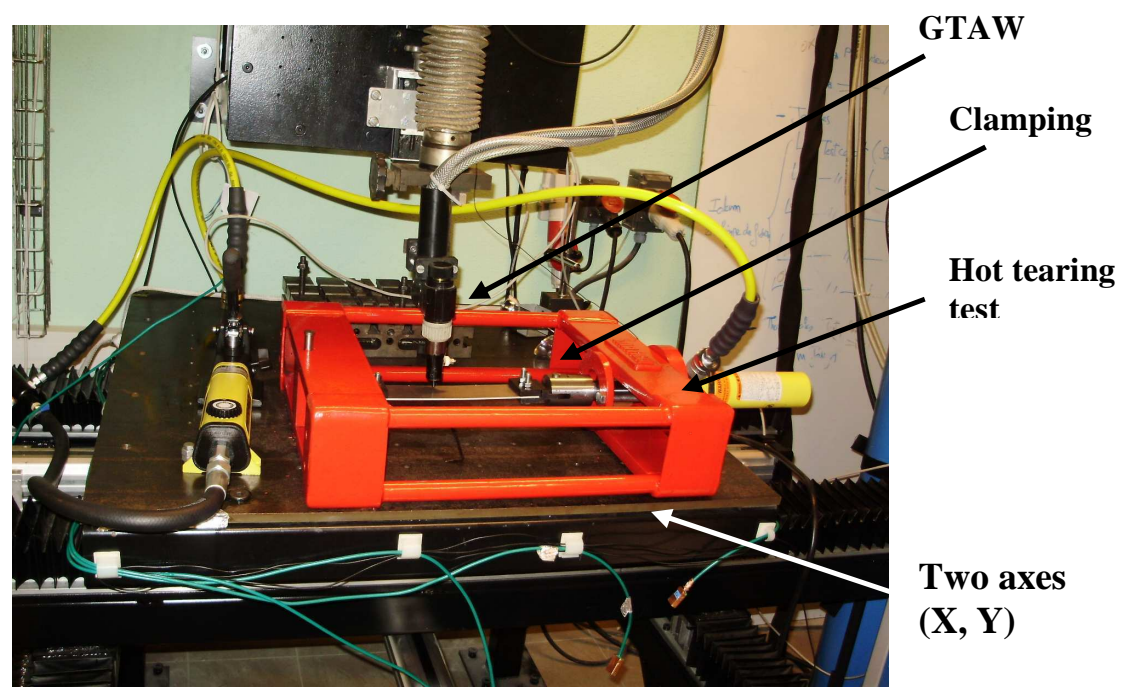

Fig. 6. Two axes table with hot tearing test.

These tests allow the cracks initiation in quasi-stationary state, in the mushy zone where complex solidification mechanisms are observed. A crack initiate, for all conditions tested, transversely to the welding direction and then propagates along the center line as shown in figure 1. Dendritic columnar grains are present at the fusion boundary, whereas dendritic equiaxed grains are visible in the center, for our welding conditions. Crack initiation occurs at the fusion boundary between the columnar grains. Crack initiation is a combination of brittle microstructure at the fusion boundary and a large tensile stress concentrated in the liquid film present between these grains. A longitudinal tensile facilitates transversal cracks initiation between columnar grains more easily than transversal tensile.

The study of complex coupling between process, material and mechanical loading requires an experimental investigation but also a numerical analysis for hot cracking study. Indeed, it is necessary to have information on the strain and stress field around the welding pool during cycle to better understand cracking mechanism.

\section{Numerical modeling}

Chihoski [4] was the first to study stress distribution around a moving localized heat input on thin sheets of aluminum alloy. The temperature 
distribution and the thermal dependent mechanical behavior of the alloy require numerical modeling to determine the stress distribution.

In addition to thermal and mechanical aspects, thermo- metallurgical issue must be solved by assessing microstructural parameters. A simulation (with Sysweld finite element software) of a weld seam in TIG process is made on a thin sheet of aluminum alloy, hence the choice of $2 \mathrm{D}$ modeling. A displacement is imposed as boundary condition to the sample before welding. This displacement gives rise to a preload stress and this displacement is determined so that the tensile loading corresponds to the yields stress of the alloy $(+200 \mathrm{MPa})$. The tensile preload and the welding direction are along y axis (figure 7). The heat source modeled as a Gaussian and welding speed is $15 \mathrm{~mm} / \mathrm{s}$. Quadrilateral finite elements are used to sheet mesh. A fine mesh (8-node (Q8)) is used in the welded region. The purpose of this section is to understand the preload influence on stress and strain fields in the mushy zone at the rear of the weld pool.

Stresses and strains are generated by external loading, thermal loading induced by welding and heterogeneity of the mechanical behavior in different areas due to thermal gradient. The thermal dependence of Young's modulus, thermal expansion coefficient and yield strength is taken into account. The temperature ranges of material properties is $0^{\circ} \mathrm{C}$ to $700^{\circ} \mathrm{C}$. The non-linearity of material properties in high temperature range is taking into account using the hot rheological data studied by Maisonette [5]. In this study, the solidification shrinkage is modeled by a tabulated law in the solidification range. The weld pool shape and size are directly related welding speed and current and sample geometry. The weld pool geometry has a strong influence on the stress and strain distribution. An elongated weld pool representative of our experimental results is simulated by adjusting the heat source parameters. The heat affected zone is best described by a mixed hardening (ratio of kinematic hardening and isotropic hardening), because of the alternate loading undergoes during welding. The dissolution of precipitates in the molten zone and the restoration of the hardening in the heat affected zone will be taken into account. The strain comparison obtained by numerical simulation with the elasto-plastic laws or elasto-visco-plastic laws having shown that very few differences, viscosity is neglected. This relatively simple model is applied to the test geometry to study the preload influence on the susceptibility to hot cracking. Strain rate, necessary to study the RDG criterion, is calculated in post processing using the simulation results. Then, this model is used to propose possible optimization of welding in order to limit crack initiation. 


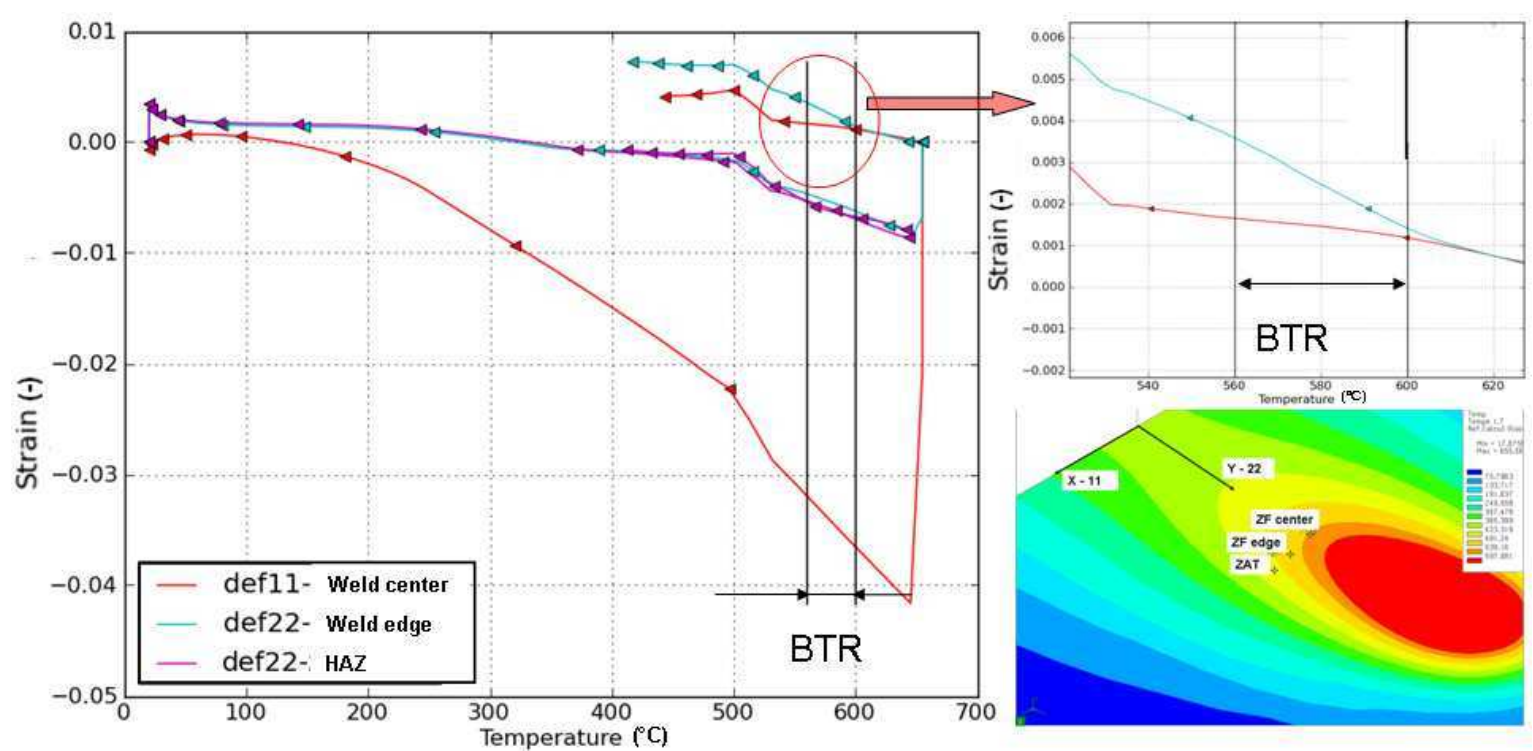

Fig.7. Strains evolution for developed test.

Figure 7 shows the strain evolution during welding and cooling in three points of the weld pool: one point in the weld center, one in the mushy zone at the fusion boundary and the last in the heat-affected zone. The normal strain $(\varepsilon 11=\varepsilon \times x)$, transverse to the weld, is shown in the weld center. For two other points, we have chosen to represent the longitudinal strain $(\varepsilon 22=\varepsilon y y)$. These points and strain direction were chosen in agreement with experimentally observed cracks location and orientation. In the BTR, represented by two vertical black lines in figure 7, the strain evolution promotes hot cracking in the weld. Indeed, the fusion boundary is subject to positive strain, mainly due to shrinkage, thus facilitating crack initiation. Moreover, results in figure 6 show a positive longitudinal strain rate at the solidification end, most important for point located at the fusion boundary, in the mushy zone.

The transverse strain (Exx) in the weld center is positive at solidification end which facilitates crack propagation. The strain at the point located at the weld center (transverse tensile) is twice lower than on the fusion boundary in the mushy zone (longitudinal tensile) in the BTR which is in accordance with experimental observations, cracks always initiate transversely, at the fusion boundary. The risk of liquation cracking is limited, as the point situated in the heat affected zone exhibit a negative strain. 


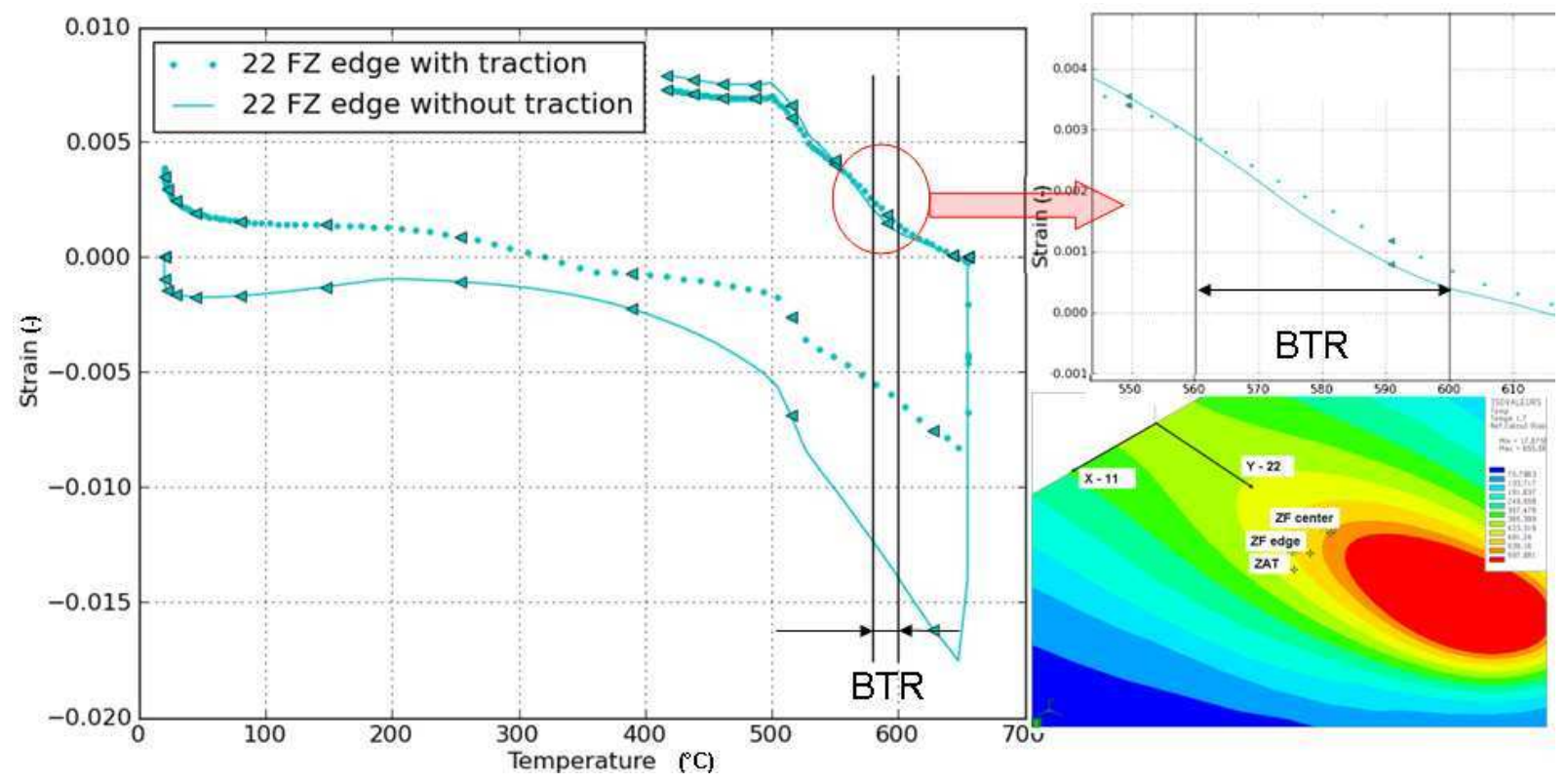

Fig.8. Influence of a pre-load stress on the longitudinal strain at the fusion boundary.

Simulation is used to show the influence of a tensile initial stress on the mushy zone. Two cases are studied here: a specimen subjected to longitudinal tensile stress and a second with zero initial stress, i.e not clamped. The longitudinal strain evolution at the fusion boundary is presented figure 8 . In the BTR, the strain is thirty percent greater for the case with traction than without. A pre-load stress exacerbates the tensile loading at the fusion boundary in the welding direction and consequently promotes hot cracking initiation.

However, mechanical loading of the sheet is not the only parameter affecting hot cracking initiation. Metallurgy plays an important role and should be considered.

\section{Experimental results}

\section{In situ observation}

High speed camera recordings of the mushy zone during welding were carried out, in order to observe crack initiation. Mushy zone observation is 
complicated by the formation of oxide at the weld pool surface. With an improved gas protection, the weld pool boundary appears as can be seen in figure 9. In-situ observations permit a better understanding of the hot tearing initiation concerning solid fractions and dendrite morphology.
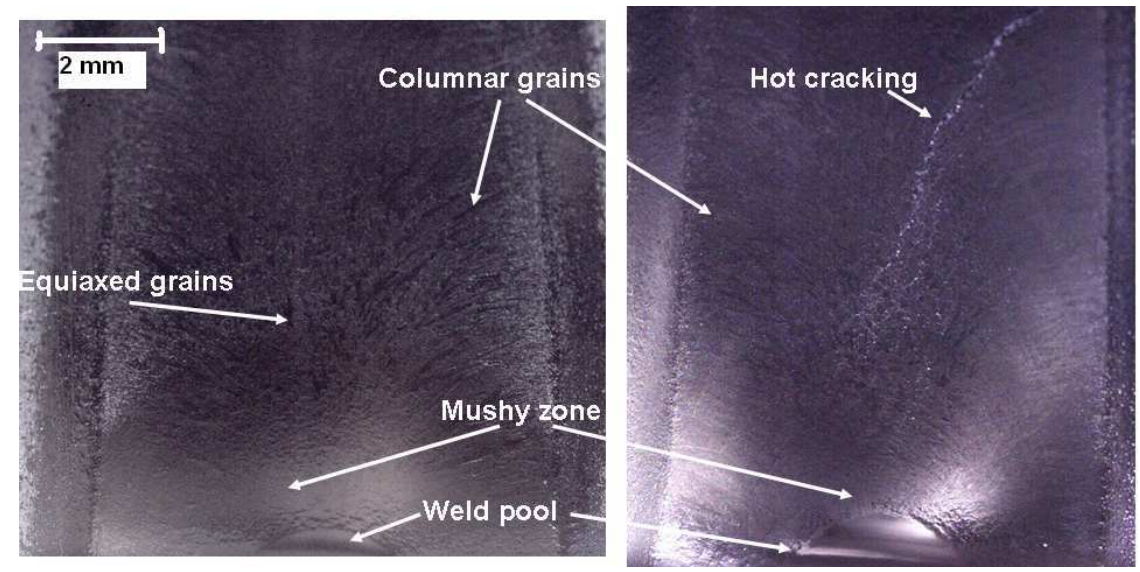

Fig.9. Video recording image during welding showing the weld pool tail.

Two areas with distinct granular structure can be observed on the in-situ recording, in the re-solidified zone: a columnar dendritic zone on the edge of fusion zone, and an equiaxe dendritic zone in the center. The solidification rate is minimal at the fusion boundary and is maximal at the center (as opposed to the thermal gradient). However, at the lowest speed welding, the fusion zone is composed entirely of columnar dendritic grains. When the welding speed becomes greater than the limit of solidification speed, equiaxed grain formation is possible. Theoretical considerations have explained these results. The formation of an equiaxe dendritic zone is promoted by high undercooling at the liquidus isotherm. Undercooling is favored by high solidification speed and low thermal gradient [3]. The thermal gradient is lower in the center of the fusion zone, which explains the nucleation of equiaxed grain in this zone.

Observation with high speed camera confirms that cracks initiate in the columnar zone, in a direction transverse to the welding direction. As a result, and depending on welding parameters, crack stop is observed in the equiaxe zone. In addition, bifurcation and a longitudinal propagation are present in the equiaxed grains zone. 


\section{Post-mortem study of crack morphology}

After welding, the samples are cut out to obtain cross section views. The microstructure is studied using a combination of optical microscopy and scanning electron microscopy.

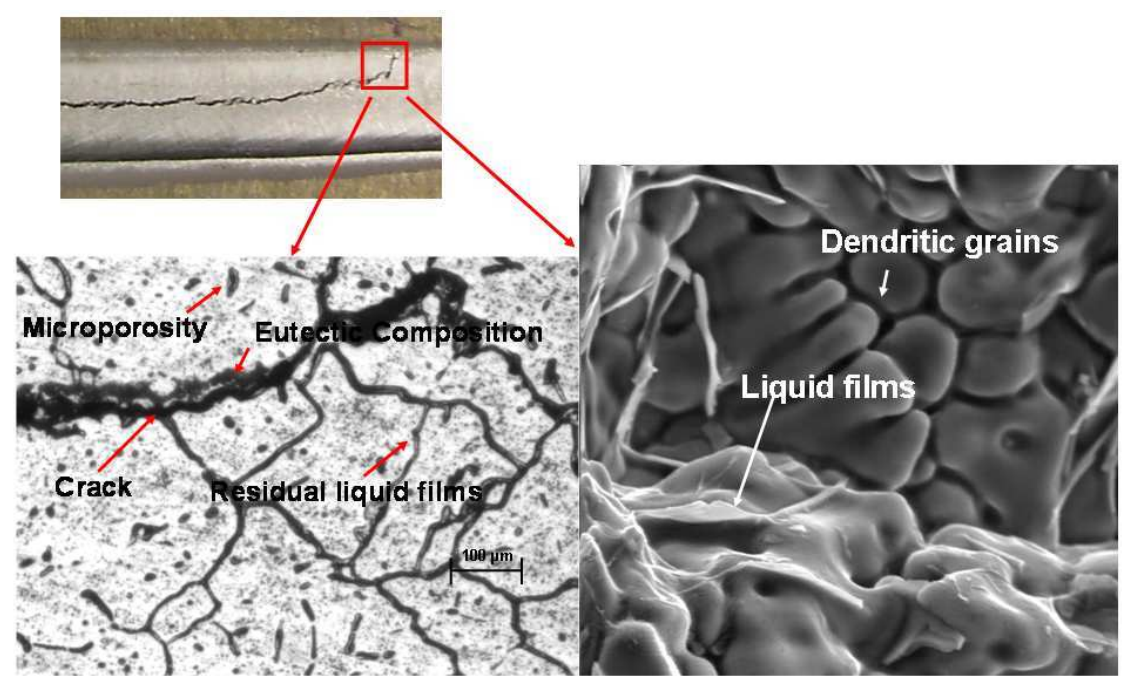

Fig.10. Observation of cracks: (a) macrograph top view, (b) micrograph in transverse section, (c) SEM fracture.

Microscopic observations of cracked areas (figure 10b), clearly show intergranular cracks generated by a debonding of liquid films. Indeed, the coalescence solid fraction, corresponding to the lower boundary of the BTR is reached first between two dendrites of the same grain, and then between two grains, which promotes the initiation of inter granular cracks rather than intragranular. The presence of liquid films at the end of solidification is visible on the fracture surfaces using scanning electron microscopy (cf. figure 10c).

The microstructure observation revealed a very characteristic grain structure, with peripherical columnar grains, oriented in a direction perpendicular to the welding direction, and central equiaxed grains, for our welding conditions [6,7]. The zone width depends on the welding parameters that vary, namely, with current and speed welding. Hot tearing sensitivity is also correlated to the grain morphology in the mushy zone. It is generally admitted that columnar dendrite morphology, with dendrites growing in the thermal gradient direction, generally observed for rather 
low solidification speed and/or high thermal gradient [3], is more sensitive than equiaxe dendric morphology. This can be understood by the fact that liquid feeding between the equiaxed grains is facilitated even at the solidification end, as well as crack healing by liquid feeding. It has been confirmed with these observations that for most of the samples, the crack initiation occurs between the dendritic columnar grains, on the weld pool edges.

This is not a surprising result, because this area combines an unfavorable microstructure, and the higher strain level, as shown in previous section. The longitudinal applied tensile preload during the test favors also transverse cracking between columnar grains.

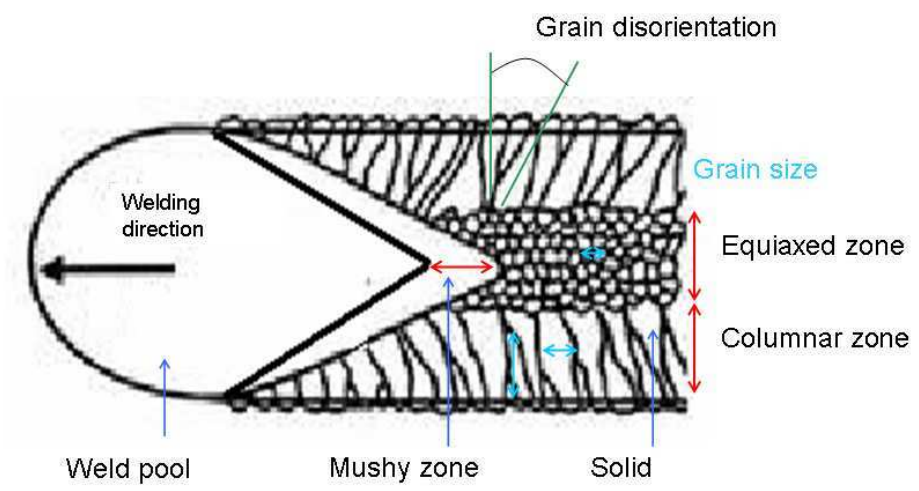

Fig.11. Microstructural characteristics in welding.

The morphology of the fusion zone is also characterized by quantitative parameters, such as grain shape and size, the growth direction and the disorientation between grains (figure 11). For instance, the emergence of columnar dendritic grains at the center line increases hot cracking sensibility, according to Hunziker [8]. If the thermal conditions leading to the formation of various grain morphologies are well known, the relationship between welding parameters and microstructure is still not fully explained, because of the complex relationships between these parameters and the temperature field.

For our tests, a columnar dendritic zone about $1,5 \mathrm{~mm}$ on each side and an equiaxe dendritic zone about $2 \mathrm{~mm}$ are found. However, the proportion of each grain morphology zone is a function of welding parameters. A correlation between the relative size of these zones and the crack propagation can be seen. When the equiaxe width zone is small compared to the total width of the cord, crack will tend to spread more 
easily in a large longitudinal crack. Moreover, when the weld seam is shifted closer to one edge, the crack always initiates from that side. A thermal asymmetry is then created in the weld bead.

In our case, the columnar grains growth direction is characterized by an angle of 80 degrees relative to the welding direction. Grain orientation also affects the ability of the liquid to feed the mushy zone. Wang showed the importance of disorientation between two grains [9]. This disorientation also limits coalescence and possible crack healing. The maximum disorientation found in our tests is about 22 degrees. However, crack initiation does not occur where the grains are highly disoriented between them in our case. The distance between arc initiation and first crack initiation appears rather constant, between 2 and $4 \mathrm{~cm}$.

\section{Influence of process parameters}

Welding parameters are classified as cracking or not cracking conditions for a given tensile initial stress. Figure 12 shows the crack sensitivity evolution of 6061 alloy as a function of welding parameters. After welding, the cracks were first revealed by dye penetrant testing. The results highlight several failure modes, depending on process parameters, figure 11. For high welding speed, some small transverse cracks are observed at the beginning of the welding line at the fusion boundary, which deviate rapidly to propagate in a large longitudinal crack (width of $0.6 \mathrm{~mm}$ on average). However, when speeds are lower, there is a succession of fine transverse cracks (less than $30 \mu \mathrm{m}$ in width) that do not reach the central equiaxe zone. For each welding speed, except the lowest, there is a critical welding current above which cracking is observed. It can be observed that large longitudinal cracks are only observed for high welding speed $(20 \mathrm{~mm} / \mathrm{s})$. 


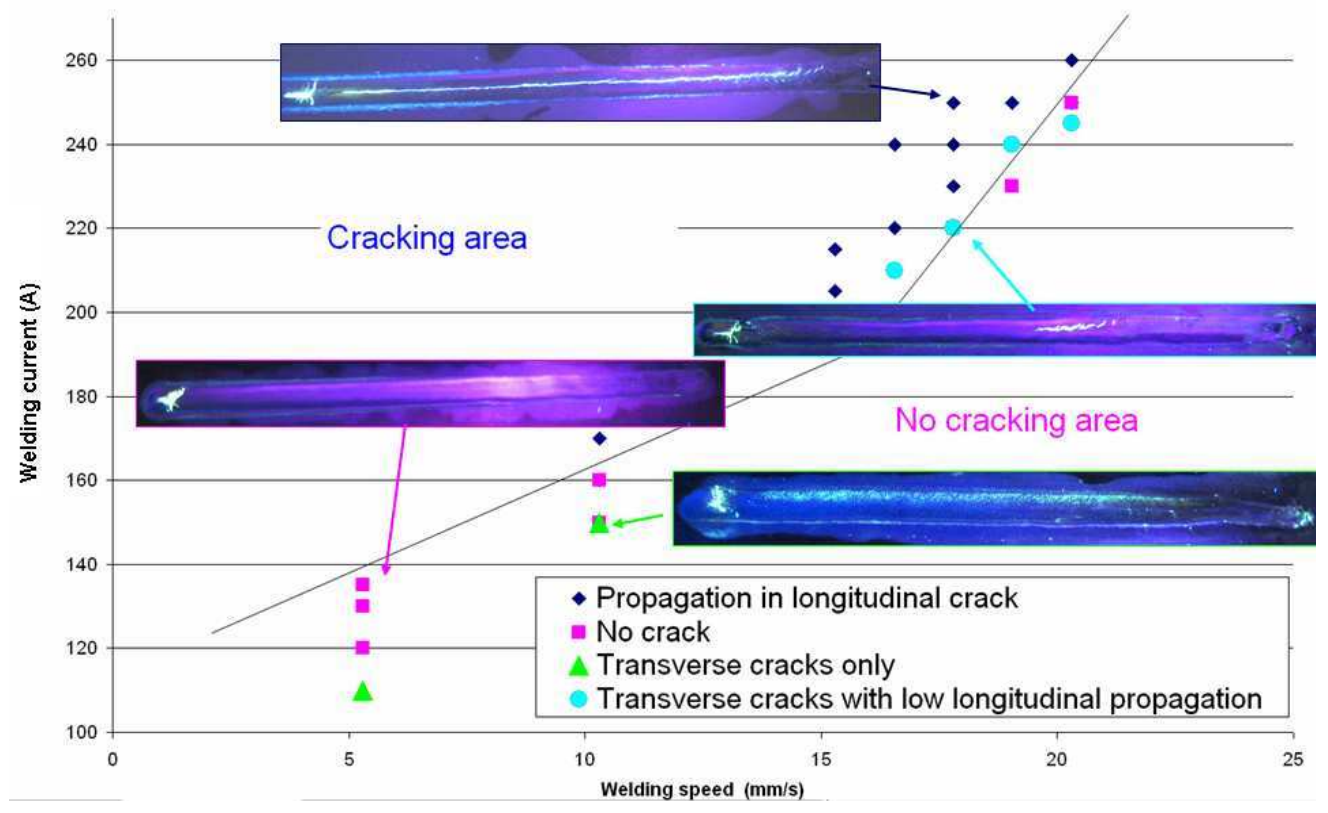

Fig.12. Hot cracking map with a pre-load stress of 200MPa.

\section{RDG criterion}

To finish this study based on pre-loading influence on hot cracking initiation, the crack initiation criterion developed by Rappaz, Drezet, Gremaud (RDG criterion) [10] has been used to compare hot cracking sensitivity. This criterion is applied as post processing of finite elements calculations presented before. The RDG criterion, based on the calculation of a critical depression $\Delta P_{\max }$ on dendrite foot requires the total strain rate of the solid skeleton perpendicular to the grain growth direction and the liquid ability to flow between grains calculated using Carman Kozeny permeability model developed for porous media. The formulas for calculating this depression are presented below (cf. eq. 1 and 2). $\mu$ and $\beta$ correspond respectively to liquid viscosity and to solidification shrinkage. The temperature $\mathrm{T}$, the temperature gradients $\mathrm{G}(\mathrm{T})$, the strain rate $\dot{\mathcal{E}}_{P}(T)$ and the solidification speed $v_{T}$ are deduced from the numerical simulation of the welding test made in parallel to experimental investigations. 


$$
\begin{aligned}
& \Delta P_{\max }=\frac{180 \cdot(1+\beta) \cdot \mu}{\lambda_{2}^{2}} \int_{T_{S}}^{T_{L}} \frac{E(T) \cdot f_{s}(T)^{2}}{\left(1-f_{s}(T)\right)^{3} \cdot G(T)} d T+\frac{180 \cdot v_{T} \cdot \beta \cdot \mu}{\lambda_{2}^{2}} \int_{T_{S}}^{T_{L}} \frac{f_{s}(T)^{2}}{\left(1-f_{s}(T)\right)^{2} \cdot G(T)} d T \\
& E(T)=\frac{1}{G(T)} \int_{T_{S}}^{T} f_{s}(T) \cdot \dot{\varepsilon}_{P}(T) d T
\end{aligned}
$$

The characteristic length $\lambda_{2}$ (spaces between the secondary dendrite arms) is extracted from the micrographs. The evolution of the solid fraction $\mathrm{fs}(\mathrm{T})$ is calculated using Scheil-Gulliver relation. The pressure evolution, calculated along dentrictic columnar grains, is shown on figure 13. The pressure drop evolution was calculated by following the red line in figure 13.
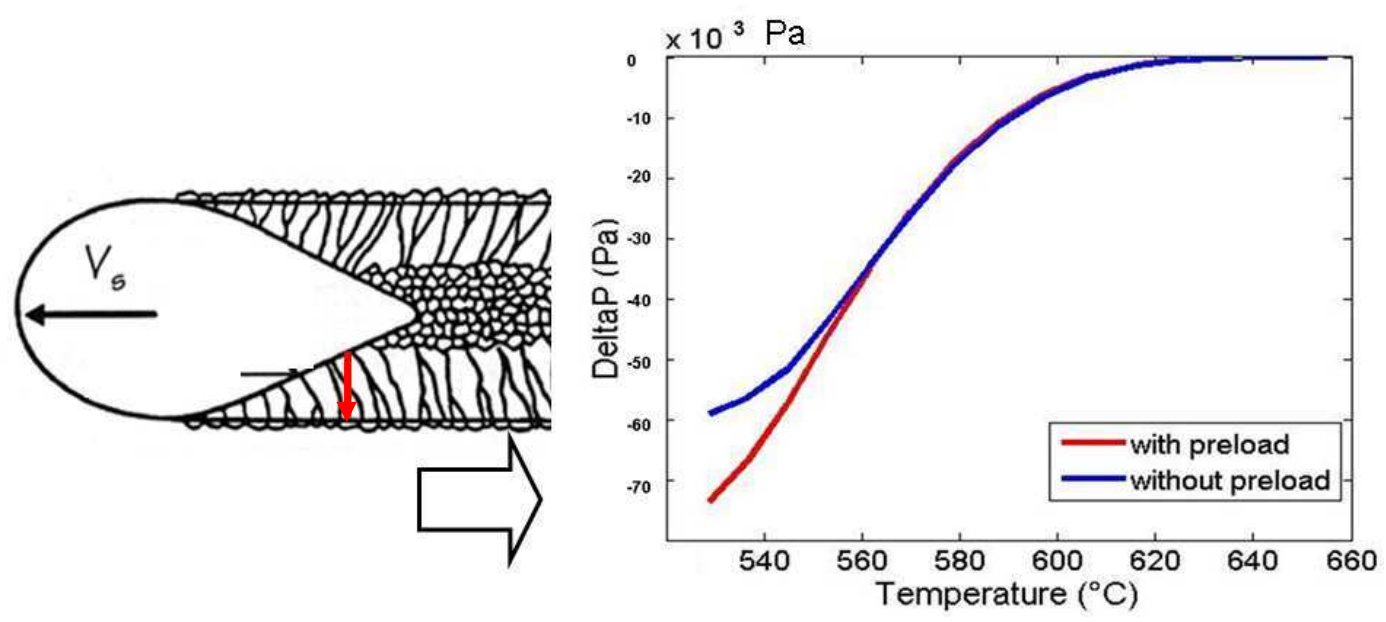

Fig.13. Influence of preload on the RDG pressure drop.

The pressure drop is greatest at the fusion boundary, near the dendrite foot where hot tear initiate. It is more important for the test with traction than the test without. This seems to confirm the influence of the tensile preload in the welding direction on the hot cracking sensitivity. The connection between the RDG pressure drop and the welding parameters allows us to highlight the metallurgical, process and mechanic interactions. 


\section{Conclusion}

It has been demonstrated that solidification cracking is a faceted problem, with many influencing parameters. Many studies examined hot tearing but few of them have studied the relationship between mechanical aspect induced by the process and microstructure. A new, original and simple test has been developed to promote hot cracking initiation. Applying a tensile preload on the welding direction promotes crack initiation for our welding conditions. Knowledge of mechanical loading imposed allows us to work on the interaction between material and process. Cracking phenomenon is the correlation of sensitive microstructure and critical mechanical loading. Cracks initiated for all our welding conditions, in the peripheral zone, which has a columnar grain structure, while the central zone of equiaxed structure is more resistant to the phenomenon.

Observation using high speed camera helps us to better understand the mechanisms of crack initiation and the bifurcation at the grain scale. Moreover, the mapping representing the field cracking based on welding conditions shows the importance of welding power on the crack initiation and the different failure types. The microstructure characteristic lengths measured are introduced in the RDG criterion in order to introduce microstructural parameters in hot cracking sensitivity.

New tests will be accomplished by varying the tensile preload in order to deepen the cracks initiation conditions in welding. In order to optimize the process, localized heating could be envisaged [10] and the microstructure of the critical zone changed. These changes will also occur on mechanical loading of the mushy zone thus revealing importance of structural effect on crack initiation. An other improvement is to modify the microstructure by acting on the process (acting to modify grain orientation, grain size, zone proportion) to reduce hot cracking.

\section{References}

1. Farrar, (2006) Hot cracking tests, Hot Cracking Phenomena in Welds, p.291304.

2. Cross, (2005), On the origin of weld solidification cracking - Hot Cracking Phenomena in Welds - p3.

3. Gaumann, (1997) Nucleation ahead of the advancing interface in directional solidification, Materials science and engineering, A226-228.

4. Chihoski, (1972), Weld cracking in aluminium alloy - Welding journal. 
5. Maisonnette (2010), Influences mécaniques et métallurgiques de procédés haute température sur un alliage d'aluminium 6061-T6 - Thèse LaMCoS.

6. DebRoy, (1995), Physical processes in fusion welding, Reviews of Modern Physics, Vol.67, No.1.

7. Norman, (1999), Effect of welding parameters on the solidification microstructure of autogenous TIG welds in an Al-Cu-Mg-Mn alloy, Materials Science and Engineering A259, p 53-64.

8. Hunziker, (2000) On formation of a centerline grain boundary during fusion welding. Acta mater. 48, 4191-4201.

9. Wang, (2004), Solidification cracking of super alloy single and bi crystals, Acta Materiala 52, 3173-3182.

10. Rappaz, Drezet, Gremaud (1999) A new hot tearing criterion, Metallurgical and materials transactions A 1999, 30A, 449.

11. Hernandez, (1984), The influence of external local heating in preventing cracking during welding of aluminium alloy sheet - Welding journal $-\mathrm{p} 84 \mathrm{~s}$. 